\title{
Elementos esenciales para el desarrollo de una estrategia fuerte de promoción de la salud: oportunidades y desafíos para maximizar el rol de las farmacias comunitarias
}

\section{Erio Ziglio}

Doctor. Profesor honorario. Health University of Applied Sciences Tyrol, Austria. Profesor visitante. Glasgow Caledonian University, Campus London, Reino Unido. Miembro de la Facultad Management Center Innsbruck, Austria. Responsable del Programa de Promoción e Inversión en Salud de la Oficina Europea de la Organización Mundial de la Salud (OMS) en Copenhague (1999-2014). En 2003 fue nombrado jefe de la Oficina Europea de Inversión para la Salud y el Desarrollo de la OMS en Venecia hasta su jubilación en 2014.

Durante 23 años, Erio Ziglio ha sido responsable del Programa de Promoción e Inversión en Salud de la Oficina Europea de la Organización Mundial de la Salud (OMS) en Copenhague. En 2003 fue nombrado jefe de la Oficina Europea de Inversión para la Salud y el Desarrollo de la OMS en Venecia hasta su jubilación en 2014

Actualmente es profesor honorario de la Health University of Applied Sciences Tyrol (Austria) y miembro de facultad en Management Center Innsbruck (Austria), donde participa en la enseñanza y capacitación de futuros gerentes y líderes que trabajan en los sectores de salud, sociales y otras políticas públicas. También forma profesionales en los campos de terapia ocupacional y gestión social. En 2018 forma parte de la Glasgow Caledonian University como profesor invitado en su sede en Londres, Reino Unido.

Además de sus más de 20 años de trabajo con la OMS, el Dr. Ziglio tiene una gran trayectoria profesional en el mundo académico, tanto en Europa como en Norteamérica, como investigador, conferencista y profesor invitado en las universidades de Edimburgo en Escocia; Toronto y Carlton-Ottawa en Canadá; o Yale en Estados Unidos.

Durante su carrera profesional, el Dr. Ziglio ha proporcionado asistencia técnica y consultorías en más de 50 países, donde se ha involucrado en formas de políticas y en el desarrollo profesional y organizacional, tanto en Europa como a nivel mundial.

El Dr. Ziglio ha dictado conferencias a nivel internacional y publicado ampliamente sobre temas de promoción de la salud, políticas y planificación de la salud, determinantes sociales de la salud, inequidades en salud y salud de la población en un marco de desarrollo y gestión sostenible.

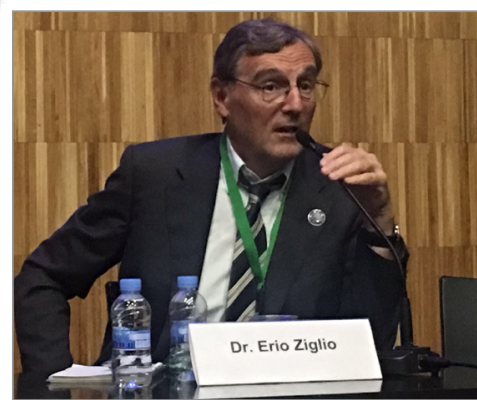

Erio Ziglio

\section{PALABRAS CLAVE}

Salud pública, promoción de la salud, farmacia comunitaria

\section{ABREVIATURAS}

OMS: Organización Mundial de la Salud

\section{KEYWORDS}

Public health, promoting health, community pharmacy

Recibido: $15 / 11 / 2019$

Aceptado: 2/12/2019

Disponible online: 30/12/2019

\section{INTRODUCCIÓN}

El 20 y el 21 de septiembre de 2018 tuve la oportunidad de facilitar una conferencia organizada por la Sociedad Española de Farmacia Familiar y Comunitaria (SEFAC) en Lanzarote, España. El título de dicha conferencia fue Salud pública y farmacia comunitaria como promotora de salud. Durante la conferencia en Lanzarote, mi objetivo fue ofrecer un marco de referencia útil para fortalecer el papel de las farmacias comunitarias dentro de una estrategia sólida de promoción de la salud relevante para el contexto de Canarias. En este artículo tocaré y expandiré algunas de las reflexiones que hice en esa conferencia, con un enfoque nacional y global.

Key elements for the development of a strong health promoting strategy: opportunities and challenges to maximize the role of community pharmacies

\section{INTRODUCTION}

On September 20 and 21, 2018, I had the opportunity to facilitate a conference organized by the Sociedad Española de Farmacia Familiar y Comunitaria [Spanish Society of Family and Community Pharmacy] (SEFAC) in Lanzarote, Spain. The title of this conference was Public Health and Community Pharmacy as Health Promoter. During the conference in Lanzarote, my objective was to offer a reference standard to strengthen the role of community pharmacies within a solid strategy of promoting health relevant to the context of the Canary Islands. In this article I will cover and explore reflections made in this conference, with a national and global focus. 


\section{Promoción de la salud: un camino iniciado desde 1986 hasta nuestros días}

En noviembre de 1986, fui uno de los expertos que se reunió en Ottawa, Canadá, para elaborar la mundialmente famosa Carta de Ottawa para la promoción de la salud. En esta carta, la promoción de la salud es definida como el proceso que permite a las personas tomar el control de los determinantes de su salud (1). En las siguientes tres décadas, la Organización Mundial de la Salud (OMS) ha organizado periódicamente conferencias globales sobre promoción de salud con el objetivo de modernizar la salud pública y ganar compromiso político para promover la salud y reducir las iniquidades en salud. La última de estas conferencias fue celebrada en 2016 en Shanghái, China (2).

Dos han sido los objetivos a lo largo de este proceso global coordinado por la Organización Mundial de la Salud. En primer lugar, asegurar que la promoción de la salud sea tomada con seriedad por autoridades internacionales, nacionales y locales, con planes de acción claros e inversión en esta área. El otro objetivo es fomentar esfuerzos políticos para posicionar la promoción de la salud como un elemento clave de desarrollo local, nacional y global dentro del marco de la Agenda 2030 y sus 17 Objetivos de Desarrollo Sostenible (3).

Sin duda, las estrategias diseñadas para promover la salud de la población y abordar las desigualdades en salud necesitan ser parte integral de las agendas de desarrollo social, económico y ambiental, a nivel nacional y local. Este es un enfoque clave para garantizar que una estrategia de promoción sea creíble y efectiva. En efecto, dicha consideración es también de crucial importancia para los esfuerzos actuales en España, así como en otros países europeos. Contar con farmacias comunitarias como uno de los socios es una estrategia clave de la promoción de la salud.

\section{Necesidad de trabajar sistemáticamente para la promoción de lá salud de la población}

La Carta de Ottawa para la promoción de la salud indica cinco principales áreas de acción:
- La elaboración de una política pública sana.

- La creación de ambientes favorables para la salud.

- El reforzamiento de la acción comunitaria.

- El desarrollo de las aptitudes personales.

- La reorientación de los servicios sanitarios (mayor prioridad para la prevención y promoción de la salud).

Para avanzar de manera concreta hacia la promoción de la salud, estas cinco áreas de acción deben llevarse a cabo de manera conjunta y sistemática. Se ha probado que lo anterior ha sido difícil de realizar en muchos países. Dicha dificultad explica la persistencia de un progreso aún lento en la promoción de la salud. La promoción de la salud solo puede ser efectiva si se persiguen en conjunto todas estas áreas. Con demasiada frecuencia, los países toman "atajos" e implementan solo programas semanales para cambiar estilos de vida individuales por medio de campañas generales e información en folletos y anuncios. A pesar de que estos esfuerzos sean importantes, tendrán poco impacto a menos que todas las áreas de acción priorizadas por la Carta de Ottawa sean tomadas en consideración seriamente.

\section{La potencial contribución de las farmacias comunitarias}

Dentro del marco sistemático descrito anteriormente, las farmacias comunitarias pueden contribuir positivamente dentro de la estrategia de promoción de la salud nacional o local. Hay diversos estudios que muestran el potencial del farmacéutico comunitario como educador sanitario y agente promotor de la salud. Muchos de estos estudios señalan los beneficios que las farmacias comunitarias pueden otorgar, particularmente dentro de la estrategia local de promoción de la salud. La mayoría de estos beneficios provienen del estrecho vínculo de las farmacias comunitarias y la población local $(4,5)$. Entre estos se encuentran:

- Fácil acceso a los servicios, ya que no es necesario contar con una cita previa y la población puede disfrutar de un horario de apertura amplio.
- Muchos miembros del personal pertenecen a la comunidad local y entienden la cultura local.

- Un número creciente de farmacias comunitarias cuentan ahora con una 'sala privada' de consulta, en donde la gente puede solicitar consejos y recibir asesoramiento.

- Muchos programas preventivos y en favor de la salud locales y nacionales cuentan con el apoyo de las farmacias comunitarias, que a menudo también desempeñan un papel de fomento de la promoción de la salud.

Existen también algunos obstáculos para el papel de las farmacias comunitarias en la promoción de la salud. Entre estos se encuentran temas relacionados con la capacitación de los farmacéuticos, donde la promoción de la salud es muy marginal o prácticamente inexistente. La promoción de la salud debe ser parte integral de los planes de estudio del Grado de los farmacéuticos. Otro obstáculo es la tendencia a que la función de asesoramiento de los farmacéuticos en la promoción de la salud tiende a ser reactiva en lugar de proactiva. Un problema más que tiene que atenderse es que, en muchas ocasiones, el rol de la promoción de la salud en las farmacias comunitarias es percibido como "intrusivo" o incluso inapropiado por otras profesiones clínicas o de la salud. Estos son desafíos que necesitan ser abordados. En ellos está la razón por la cual los farmacéuticos tienden a concentrarse en el uso adecuado de medicamentos recetados y no tanto en cuestiones que apoyan a los ciudadanos en una visión más integral de la salud.

Abordar estos desafíos aumentará la credibilidad de las farmacias comunitarias en la promoción de la salud, incluso en programas en donde ya están jugando un papel. Algunos de estos programas son los siguientes:

- Programas para dejar de fumar.

- Cáncer de piel.

- Conocimiento del asma.

- Programas para perder peso.

- Apoyo a la salud mental.

- Apoyo para la recuperación del uso indebido de drogas.

- Asesoramiento, educación o apoyo a grupos de alto riesgo.

- Una amplia gama de factores de riesgo para enfermedades no transmisibles. 
Por lo tanto, no cabe duda de que las farmacias comunitarias tienen un fuerte papel en el desarrollo de métodos y habilidades para apoyar el cambio de comportamientos que dañan a la salud. De igual forma, es altamente deseable explorar más a fondo el papel de las farmacias comunitarias en las intervenciones para reducir las desigualdades en salud dentro del contexto poblacional que operan.

De la misma manera, la función de promoción que las farmacias comunitarias pueden jugar es aún muy poco utilizada. Así, las farmacias comunitarias pueden jugar un papel mucho más importante en la promoción de la protección de la salud contra los riesgos y peligros que amenazan a una población determinada. Las farmacias comunitarias podrian defender la búsqueda de un entorno físico y social que promueva la salud. Todo esto aumentaría la credibilidad de las farmacias comunitarias en la promoción de la salud.

\section{Conclusiones}

Se espera que esta breve contribución pueda ayudar a crear un impulso para una estrategia sólida de promoción de la salud en donde las farmacias comunitarias puedan desempeñar un papel eficaz. Tal papel debe verse en cooperación, coordinación e inte- gración de esfuerzos con otros socios institucionales. Dentro de dicha estrategia, las farmacias comunitarias pueden jugar un papel muy importante. Para obtener resultados tangibles de promoción de la salud, las farmacias comunitarias deberán fomentar alianzas con otros componentes del sistema de salud pública y abogar por la salud de la población, así como por la reducción de las desigualdades en salud y por una participación activa de los actores sociales. Sin ese enfoque estratégico, las farmacias comunitarias seguirán siendo un elemento marginal a pesar de los proyectos e iniciativas apreciables que se están desarrollando tanto en España como en otros países alrededor del mundo.

\section{Reconocimiento}

Doy el debido reconocimiento a Alejandra Tlacuilo Morales, licenciada en ciencias farmacéuticas, por el apoyo recibido al revisar el texto de este artículo.

\section{Referencias bibliográficas}

1. Carta de Ottawa para la Promoción de la Salud [Monografía en internet] Organización Mundial de la Salud. 1986 Ginebra. [Último acceso 19/12/19] https://www.paho.org/tierra/index. php/subject-matters/documents/carta-ottawa-promocion-salud\#
2. Declaración de Shanghai sobre la Promoción de la Salud [Monografía en internet] Organización Mundial de la Salud. 2016 Ginebra. [Último acceso 19/12/19] https://www.who.int/healthpromotion/conferences/9gchp/shanghai-declaration/es/

3. Transformar nuestro mundo: La Agenda 2030 para el desarrollo sostenible [Monografía en internet] Cumbre para el desarrollo sostenible. Naciones Unidad. Nueva York 2015. [Último acceso 19/12/19] https://www.google. com/url? sa =t\&trct=j\&tq =Etesrc $=$ stt source $=$ web\&tcd $=10$ ctcad $=$ rjactuac $\mathrm{t}=8 \mathrm{Ctved}=2$ ahUKEwihvuaE0sHmAhXOC-wKHWYlA3wQFjAJegQIARAHCturl=https $\% 3 \mathrm{~A} \% 2 \mathrm{~F} \% 2 \mathrm{Fw} w$ w. ar.undp.org $\% 2 \mathrm{Fcontent} \% 2 \mathrm{Fdam} \%$ 2Fargentina\%2FPublications\%2FAgenda2030\%2FPNUDArgent-DossierODS. pdfctusg=A0vVaw0Z76fjdWgu9E0yC qkzZSzh

4. Making Every Contact Count (MECC): Consensus statement. [Monografia en internet] Public Health England, NHS England and Health Education England. Londres. 2016. [Último acceso 19/12/2019] https://www.england.nhs. uk/publication/making-every-contact-count-mecc-consensus-statement/

5. Community pharmacy offer for improving the public's health: a briefing for local government and health and wellbeing boards. [Monografia en internet] Public Health England. Londres 2016. [Último acceso 19/12/19] https://www. gov.uk/government/publications/community-pharmacy-offer-for-improving-the-publics-health 